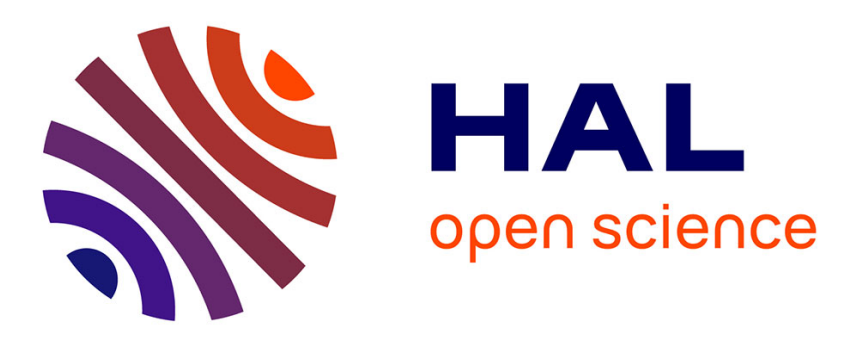

\title{
Anisotropic longitudinal electronic relaxation affects DNP at cryogenic temperatures
}

E. M. M. Weber, H. Vezin, G. Kempf, G. Bodenhausen, Daniel Abergel, D.

Kurzbach

\section{- To cite this version:}

E. M. M. Weber, H. Vezin, G. Kempf, G. Bodenhausen, Daniel Abergel, et al.. Anisotropic longitudinal electronic relaxation affects DNP at cryogenic temperatures. Physical Chemistry Chemical Physics, 2017, 19 (24), pp.16087 - 16094. 10.1039/C7CP03242K . hal-01552980

\section{HAL Id: hal-01552980 \\ https://hal.sorbonne-universite.fr/hal-01552980}

Submitted on 3 Jul 2017

HAL is a multi-disciplinary open access archive for the deposit and dissemination of scientific research documents, whether they are published or not. The documents may come from teaching and research institutions in France or abroad, or from public or private research centers.
L'archive ouverte pluridisciplinaire HAL, est destinée au dépôt et à la diffusion de documents scientifiques de niveau recherche, publiés ou non, émanant des établissements d'enseignement et de recherche français ou étrangers, des laboratoires publics ou privés. 
Cite this: Phys. Chem. Chem. Phys., 2017, 19, 16087

Received 16th May 2017, Accepted 2nd June 2017

DOI: $10.1039 / c 7 c p 03242 k$

rscli/pccp

\section{Anisotropic longitudinal electronic relaxation affects DNP at cryogenic temperatures}

\author{
E. M. M. Weber, ${ }^{a b}$ H. Vezin, (D) ${ }^{c}$ J. G. Kempf, (D) ${ }^{d}$ G. Bodenhausen, (D) ${ }^{a b}$ \\ D. Abergél ${ }^{* a b}$ and D. Kurzbach (D)*ab
}

We report the observation of anisotropic longitudinal electronic relaxation in nitroxide radicals under typical dynamic nuclear polarization conditions. This anisotropy affects the efficiency of dynamic nuclear polarization at cryogenic temperatures of $4 \mathrm{~K}$ and high magnetic fields of $6.7 \mathrm{~T}$. Under our experimental conditions, the electron paramagnetic resonance spectrum of nitroxides such as TEMPOL (4-hydroxy-2,2,6,6-tetramethylpiperidin1-oxyl) is only partly averaged by electronic spectral diffusion, so that the relaxation times $T_{1 \mathrm{e}}(\omega)$ vary across the spectrum. We demonstrate how the anisotropy of $T_{1 \mathrm{e}}(\omega)$ can be taken into account in simple DNP models.

\section{Introduction}

Dynamic nuclear polarization (DNP) allows one to obtain high polarization levels of nuclear spins in the presence of paramagnetic agents by combining electron paramagnetic resonance (EPR) with nuclear magnetic resonance (NMR). This phenomenon has led to the development of various techniques to overcome one of the major limitations of NMR, namely the intrinsically low sensitivity. ${ }^{1-3}$ The irradiation of unpaired electron spins by microwaves eventually leads to the enhancement of the nuclear polarization, most efficiently for protons (for a review see Abragam and Goldman ${ }^{1}$ ). Polarization levels $P_{\mathrm{n}}\left({ }^{1} \mathrm{H}\right)>90 \%$ can be achieved at cryogenic temperatures between 1.2 and $4.2 \mathrm{~K}^{4-6}$ In our laboratory, we often polarize heteronuclei $\left(\mathrm{X}={ }^{13} \mathrm{C},{ }^{15} \mathrm{~N}\right.$ etc. $)$ by using ${ }^{1} \mathrm{H}-\mathrm{X}$ cross-polarization $(\mathrm{CP}) .{ }^{2,7}$

The mechanisms that are responsible for the transfer of polarization from electrons to protons are complex and depend on many factors, including the type and concentration of monoor bi-radicals used for DNP, the field strength, temperature, solvent, and sample heterogeneity. Typically, one distinguishes between different models to describe DNP in the solid state under varying conditions. The most prominent examples are the solid effect (SE), the cross effect (CE), and thermal mixing (TM). ${ }^{1,5}$

TM-based hyperpolarization processes can occur when the EPR linewidth of the radical exceeds the nuclear Larmor frequency. In this case, electronic and nuclear spins are

\footnotetext{
${ }^{a}$ Département de Chimie, Ecole Normale Supérieure, PSL Research University, UPMC Univ Paris 06, CNRS, Laboratoire des Biomolécules (LBM), 24 rue Lhomond, 75005 Paris, France.E-mail: kurzbach@ens.fr, daniel.abergel@ens.fr ${ }^{b}$ Sorbonne Universités, UPMC Univ Paris 06, Ecole Normale Supérieure, CNRS, Laboratoire des Biomolécules (LBM), Paris, France

${ }^{c}$ Université de Lille1, CNRS, Laboratoire de Spectrochimie Infrarouge et Raman (LASIR), Lille, France

${ }^{d}$ Bruker BioSpin, 15 Fortune Drive, Billerica, MA 01821, USA
}

associated with distinct heat reservoirs that are characterized by distinct spin temperatures. Due to the presence of dipoledipole couplings between the electrons, one should distinguish Zeeman and non-Zeeman electron reservoirs. Only the latter is coupled to the nuclear Zeeman bath via hyperfine interactions. Microwave irradiation induces a coupling and dynamic cooling of the two electron baths, which eventually leads to a cooling of the nuclear Zeeman bath in cases where electronic spectral diffusion is fast.

The CE mechanism that underlies TM-based DNP is most efficient if two distinct EPR transitions feature a difference in resonance frequencies that matches the NMR transition. It is therefore frequently observed in the presence of bi-radicals but does not (in contrast to TM) require fast spectral diffusion.

The SE requires EPR linewidths that are narrow with respect to the NMR Larmor frequency. This condition enables a flow of polarization from the electron spins to the nuclei via forbidden flip-flop transitions driven by the pseudo-secular part of the electron-nuclear hyperfine interaction. ${ }^{8}$

In this contribution, we investigate the role of the electronic longitudinal relaxation time $T_{1 \mathrm{e}}(\omega)$, which is an important parameter for almost all DNP models. We investigate this parameter for TEMPOL (4-hydroxy-2,2,6,6-tetramethylpiperidin-1oxyl) nitroxide radicals, which feature very broad EPR spectra, where, in addition to anisotropic $g$-tensors that describe the resonance frequencies of the different spectral components, the linewidth is partly due to hyperfine interactions involving the ${ }^{14} \mathrm{~N}$ nuclei of the NO moiety. Despite recent developments of sophisticated models and extensive experimental studies ${ }^{9-15}$ of nitroxide-based DNP, the anisotropy of electronic longitudinal relaxation has so far been neglected, because it was assumed that fast electronic spectral diffusion (eSD) effectively averages $T_{1 \mathrm{e}}(\omega)$ across the entire EPR spectrum. However, under our experimental conditions we predict an incomplete averaging and show the 
impact of experimentally measured $T_{1 \mathrm{e}}(\omega)$ variations across the EPR spectrum. We demonstrate how anisotropic $T_{1 \mathrm{e}}(\omega)$ can be incorporated into simple models for DNP. ${ }^{16}$ Moreover, $T_{1 \mathrm{e}}(\omega)$ critically depends on the solvents used to form the glassy DNP matrix at low temperatures. Understanding these effects allows one to optimize DNP protocols and to shed new light on the connections between DNP enhancements and properties of EPR spectra.

\section{Materials and methods}

\section{DNP}

Data were acquired on a Bruker prototype system operating at 6.7 T ( ca. $285 \mathrm{MHz}$ Larmor frequency for protons and $188.2 \mathrm{GHz}$ for electrons) equipped with doubly tuned radio frequency (rf) coils immersed in liquid helium. All experiments were performed at $4.2 \mathrm{~K}$. To saturate the EPR transitions, continuous microwave $(\mu \mathrm{w})$ irradiation was applied between 187.6 and $188.5 \mathrm{GHz}$, with a transmitted power of about $12 \mathrm{~mW}$ at the position of the sample, generated by a $94.1 \mathrm{GHz}$ ELVA-1 source combined with a Virginia Diodes frequency doubler. The $\mu \mathrm{w}$ frequency was modulated by a $2 \mathrm{kHz}$ saw-tooth signal over a range of $\pm 10 \mathrm{MHz} .^{17,18}$

\section{EPR}

X-band CW EPR measurements at $6 \mathrm{~K}$ were performed in Lille on a Bruker ElexSys 580 spectrometer equipped with a Bruker Flexline split ring (9.4-9.7 GHz near $0.335 \mathrm{~T})$. Field-swept continuous-wave spectra were obtained by modulating the $B_{1}$ field at $100 \mathrm{kHz}$ in combination with phase-sensitive detection. The resulting spectra thus appear as first derivatives of the absorption line, not to be confused with dispersion spectra.

The field-dependence of the longitudinal electron relaxation times $T_{1 \mathrm{e}}(\omega)$ was measured at $6 \mathrm{~K}$ at Lille on the same X-band spectrometer and at ETH on a Bruker W-band ElexSys E680 EPR spectrometer ( $c a .94 \mathrm{GHz}$ at $c a .3 .3 \mathrm{~T}$ ). Echo-detected inversionrecovery experiments were performed with 200 or 400 ns echo delays, recovery delays of $0-0.5 \mathrm{~s}$ and a recycling delay of $0.51 \mathrm{~s}$ at $\mathrm{X}$-band and 0-1 s and $1.12 \mathrm{~s}$ at $\mathrm{W}$-band, respectively. Selective inversion pulses between 16 and $20 \mathrm{~ns}$ (depending on the radical concentration) length were used to excite only a narrow frequency band within the EPR spectrum, covering a width of approximately 1.8-2.3 mT.

\section{Sample preparation}

For DNP experiments, the samples were loaded in cylindrical cups made of polytetrafluoroethylene (PTFE), a material that does not contain any protons and therefore does not contribute to background signals. Sample A consisted of a $25 \mathrm{mM}$ solution of paramagnetic 4-hydroxy-2,2,6,6-tetramethylpiperidine-1-oxyl (TEMPOL) in a mixture of glycerol- $\mathrm{d}_{8}(50 \%), \mathrm{D}_{2} \mathrm{O}(40 \%)$ and $\mathrm{H}_{2} \mathrm{O}$ (10\%). The glass-forming agent glycerol prevents crystallization at low temperatures. Sample B consisted of a $25 \mathrm{mM}$ TEMPOL solution in $90 \%$ ethanol- $\mathrm{d}_{6}$ and $10 \%$ non-deuterated ethanol.

For EPR experiments, samples A and B were loaded in quartz tubes with 4 and $1 \mathrm{~mm}$ inner diameters for $\mathrm{X}$-band and $\mathrm{W}$-band experiments, respectively. The concentration was varied between
1 and $50 \mathrm{mM}$ for $T_{1 \mathrm{e}}(\omega)$ measurements (indicated in the text), for CW EPR at X-band concentrations of $1 \mathrm{mM}$ were used.

\section{Data analysis}

All NMR data were processed with NMRPipe. ${ }^{19}$ The free induction decay signals were zero filled, but not apodized to retain the natural line shapes. After Fourier transformation, the spectra were fitted to theoretical models with home-written scripts using MATLAB. CW- and absorption mode EPR spectra were simulated using the well-established EasySpin package. ${ }^{20}$

\section{Experimental design}

One of our objectives was to rationalize "proton DNP profiles", i.e., the dependence of the proton polarization $P\left({ }^{1} \mathrm{H}\right)$ on the microwave irradiation frequency. The microwave frequency was incremented in steps of $20 \mathrm{MHz}$ between 187.580 and $188.460 \mathrm{GHz}$ over a range of $880 \mathrm{MHz}$, limited by the sweep width of our microwave source. The full EPR spectrum extends over ca. $1000 \mathrm{MHz}$ approximately from $187.6 \mathrm{GHz}$ to $188.6 \mathrm{GHz}$. At each applied $\mu \mathrm{w}$ frequency, a $20 \mathrm{MHz}$ wide spin packet was saturated through microwave frequency modulation. Spectral diffusion causes this saturation to propagate partly to neighbouring spins packets, as discussed below.

For each microwave frequency, the build-up of the proton signal, which is proportional to the proton polarization $P\left({ }^{1} \mathrm{H}\right)$, was detected every $5 \mathrm{~s}$ using $1^{\circ}$ flip angle rf pulses.

Typically, the build-up of the proton polarization occurs within a few minutes when saturating near the centre of the EPR spectrum, but it can be much slower (hours) near the edges of the EPR spectrum, where the populations of the irradiated spin packets are small. It is therefore cumbersome to follow the complete build-up of the proton polarization all the way up to its steady-state at every microwave frequency. However, to a good approximation, the polarization build-up is monoexponential at all microwave frequencies. Hence, we monitored each build-up curve over a sufficiently long period (up to $\sim 50 \%$ of its plateau) to allow extrapolation to the steadystate. This is illustrated in Fig. 1a for sample A at $4 \mathrm{~K}$ near
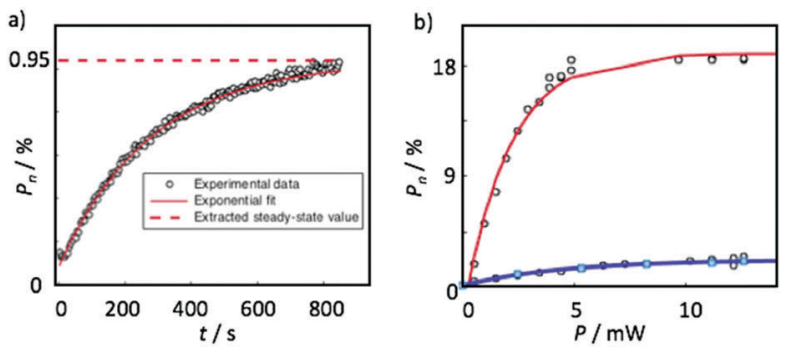

Fig. 1 (a) Initial build-up of nuclear polarization as a function of time when saturating the low-field edge of the EPR spectrum (near $187.6 \mathrm{GHz}$ ). The experimental data has been fitted to a mono-exponential function (red). The dashed line indicates the steady-state nuclear polarization estimated by extrapolation. (b) ${ }^{1} \mathrm{H}$ nuclear polarization as a function of microwave power applied at the centre of the EPR spectrum (red, 187.9) or at its outermost low-field edge (blue, $187.6 \mathrm{GHz}$ ). Above $12 \mathrm{~mW}$ of transmitted power no significant change can be observed, indicating that the corresponding bands in the EPR spectrum are fully saturated. 
the low-field $(187.6 \mathrm{GHz})$ edge of the EPR spectrum. Fig. 1b shows that the measured proton polarization, does not change when the transmitted microwave power is increased beyond $12 \mathrm{~mW}$. This is observed both in the centre and at an edge of the EPR line, indicating that the irradiated spin packets are always fully saturated in our experiments. Hence, slow build-ups near the edges do most-likely not stem from incomplete saturation.

\section{Results and discussion}

To predict the nuclear polarization with various DNP models one needs to record the EPR spectrum, which was not possible with our apparatus at $4 \mathrm{~K}$ and $6.7 \mathrm{~T}$ (centre frequency: $188.2 \mathrm{GHz}$ ). For the case at hand, we therefore simulated the relevant EPR line shape, using parameters determined at $6 \mathrm{~K}$ and $9.4 \mathrm{GHz}$ (at X-band, centre field: $0.335 \mathrm{~T}$ ). Simulations of the EPR spectra take into account both the anisotropic $g$-, and $A$-tensors of TEMPOL radicals, determined with the EasySpin routines developed by Stoll et al. ${ }^{20,21}$ The homogeneous line width as well as weak hyperfine and dipolar couplings were accounted for by a mixed Voigtian convolution. Our simulations led to the following EPR parameters for sample A (water-glycerol matrix): $A_{x x}=19.8 \mathrm{MHz}, A_{y y}=22.1 \mathrm{MHz}, A_{z z}=$ $108 \mathrm{MHz}, g_{x x}=2.00908, g_{y y}=2.00554, g_{z z}=2.00233$. For sample B ( $90 \%$ ethanol- $\mathrm{d}_{6}$ and $10 \%$ protonated ethanol), we found $A_{x x}=$ $19.9 \mathrm{MHz}, A_{y y}=25.2 \mathrm{MHz}, A_{z z}=98.1 \mathrm{MHz}, g_{x x}=2.01090$, $g_{y y}=2.00676, g_{z z}=2.00330$. These parameters determined at a concentration of $1 \mathrm{mM}$ TEMPOL allowed us to simulate EPR spectra that are relevant for our DNP experiments at $4 \mathrm{~K}$ and 6.7 $\mathrm{T}$ (centre frequency: $188.2 \mathrm{GHz}$ ), as shown in Fig. $2 \mathrm{~b}$. This absorption-mode spectrum corresponds to the integral of the derivative spectrum in Fig. 2a. Fig. 2c illustrates how different directions $(x, y, z)$ of the principal axis system (PAS) correspond to
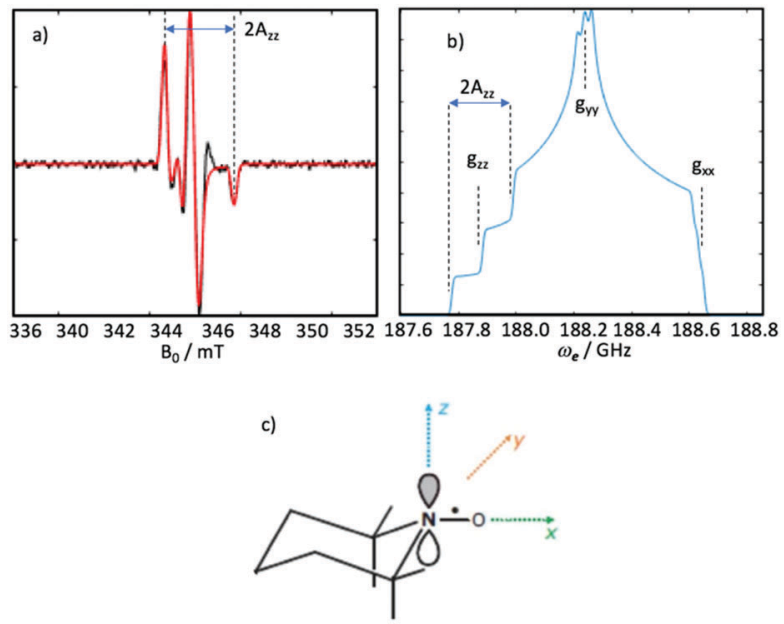

Fig. 2 (a) Experimental (black) field-swept derivative EPR spectrum of $1 \mathrm{mM}$ TEMPOL in a water/glycerol mixture at $6 \mathrm{~K}$ and $9.6 \mathrm{GHz}$ (X-band). The simulated spectrum is superimposed and depicted in red. (b) Simulated absorption line shapes at 6.7 T where the DNP experiments were carried out, using parameters obtained from the fit in (a). (c) Molecular frame and principal axis system for nitroxides. the molecular frame of TEMPOL. The values obtained by spectral simulations match published data. ${ }^{22}$

\section{Electron spin relaxation times}

For both samples A and B, field-dependent electron relaxation times $T_{1 \mathrm{e}}(\omega)$ have been determined at $6 \mathrm{~K}$ at $\mathrm{W}$-band $(94.1 \mathrm{GHz}$, centre field $3.35 \mathrm{~T}, 1 \mathrm{mM}$ TEMPOL) at selected field positions corresponding to different spin packets, as indicated in Fig. 3 together with the measured relaxation times $T_{1 \mathrm{e}}(\omega)$. The latter are also listed in Table 1.

Clearly, the relaxation times $T_{1 \mathrm{e}}(\omega)$ vary significantly across the EPR line shape, by more than an order of magnitude.

The value of $T_{1 \mathrm{e}}(\omega)$ generally depends on the coupling of the electrons to the phonons in the solid matrix in which the radicals are dispersed. For nitroxides, this coupling is generally mediated via the spin-orbit coupling of the unpaired electrons. ${ }^{8}$ The origin of the non-uniformity of the $T_{1 \mathrm{e}}(\omega)$ relaxation times of the electrons can thus be ascribed to the electronic orbitals of the electrons (the singly occupied $\pi^{*}$ molecular orbital (SOMO) in NO moieties) which are intrinsically anisotropic, leading to orientation-dependent couplings of the SOMO to the phonons. Additionally, orientation-dependent dipolar couplings can also play a minor role. We will refer in the following to this nonuniform relaxation as "anisotropic" $T_{1 \mathrm{e}}(\omega)$.

The values in Fig. 3 have been determined at a TEMPOL concentration of $1 \mathrm{mM}$. With respect to DNP where we use much higher concentrations it is important to note that we observed an anisotropic behaviour of $T_{1 \mathrm{e}}(\omega)$ at $6 \mathrm{~K}$ up to TEMPOL concentrations of $50 \mathrm{mM}$ at W-band of $94.1 \mathrm{GHz}$ (for the principle components of the $g$-tensor we found, $g_{x x}$ : $\left.T_{1 \mathrm{e}}(\omega)=52.17 \mathrm{~ms}, g_{y y}: T_{1 \mathrm{e}}(\omega)=14.99 \mathrm{~ms}, g_{z z}: T_{1 \mathrm{e}}(\omega)=63.01 \mathrm{~ms}\right)$. We observed comparable, anisotropic relaxation times of $T_{1 \mathrm{e}}(\omega)=70,39$ and $31 \mathrm{~ms}$ for $M_{\mathrm{I}}\left({ }^{14} \mathrm{~N}\right)=+1,0$ and -1 , respectively, at X band $(9.4 \mathrm{GHz}$, centre field $0.335 \mathrm{~T}, 50 \mathrm{mM}$ TEMPOL), indicating that the relaxation times do not strongly depend on the field. We shall assume that this also holds for our DNP experiments that have been performed at approximately twice the field used in W-band EPR studies. Furthermore, similar anisotropic behaviour was observed at higher temperatures of $100 \mathrm{~K}$. Our results are in agreement with the observations of Eaton, Eaton and co-workers. ${ }^{35}$ Who reported similar anisotropic relaxation for nitroxides between 20 and $150 \mathrm{~K}$.

\section{Electronic spectral diffusion}

The mechanisms of nitroxide-based DNP have been investigated in detail by Griffin, Wenckebach, Vega and their co-workers ${ }^{9,14,23-27}$ who discussed in some detail the influence of electronic spectral diffusion (eSD) on nitroxide DNP, which spreads the polarization of a spin packet across the EPR spectrum via flip-flops of dipolarcoupled electrons, thereby averaging relaxation times. If eSD were infinitely fast, we would thus observe a single isotropic $T_{1 \mathrm{e}}(\omega)$ value regardless of the saturated spin packet. However, for moderate eSD coefficients, variations in anisotropic longitudinal relaxation times $T_{1 \mathrm{e}}(\omega)$ are not completely averaged out. Thus, the interplay between eSD and the intrinsic electron longitudinal relaxation times (i.e., $T_{1 \mathrm{e}}(\omega)$ at infinite dilution) determines the spectral window 

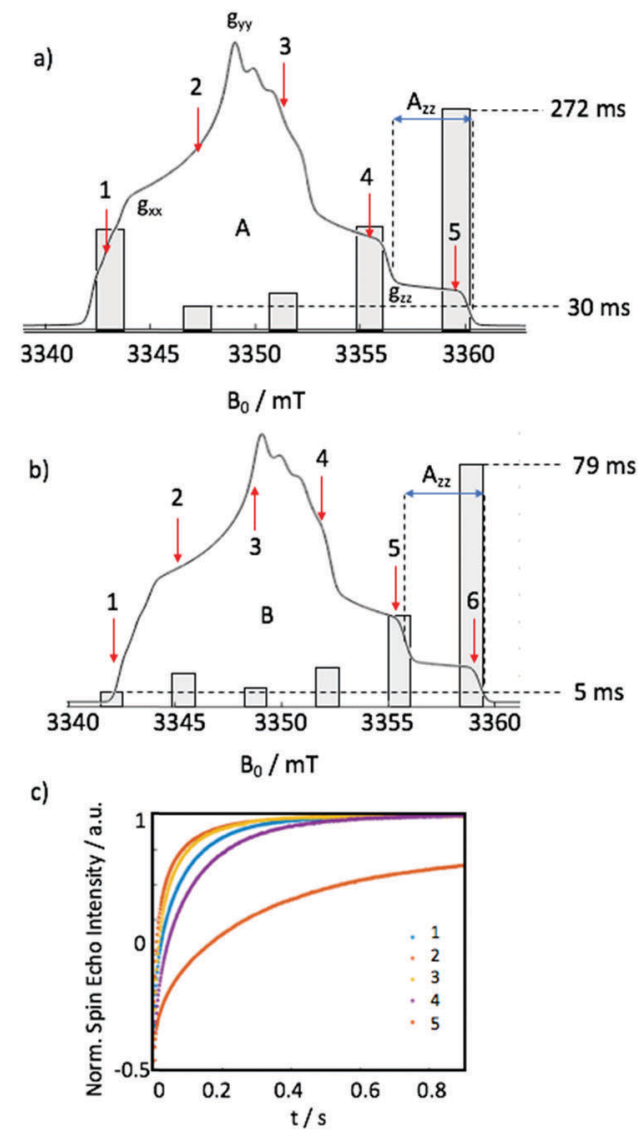

Fig. 3 ( $a$ and b) Simulated field-swept spin-echo W-band absorptionmode EPR spectrum for sample $A$ and $B$ highlighting the positions for which the longitudinal electron relaxation times $T_{1 \mathrm{e}}(\omega)$ were determined as indicated by red arrows. The heights of the grey bars in ( $a$ and b) are proportional to the $T_{1 \mathrm{e}}(\omega)$, values listed in Table 1. (c) Electron inversion recovery experiment recorded at $94.1 \mathrm{GHz}$ using electron spin echoes for sample A for the five different field positions indicated in (a). The anisotropic behaviour is clearly observable. Note that nitroxides typically feature a stretched exponential decay functions. $T_{1 \mathrm{e}}(\omega)$ values were determined by fitting to a stretched exponential function and determining the average decay rate by assuming $s\left(T_{1 \mathrm{e}}(\omega)\right) / s(0)=\mathrm{e}^{-1}$.

Table 1 Left: $T_{1 \mathrm{e}}\left(\Delta_{i}\right)$ values found for the five positions indicated in Fig. 3 for sample A. Right: $T_{1 \mathrm{e}}\left(\Delta_{i}\right)$ values found at six field positions indicated in Fig. 3 for sample B. All values were determined at $\mathrm{W}$-band $(94.1 \mathrm{GHz}$, centerfield 3.35 T, 1 mM TEMPOL)

\begin{tabular}{|c|c|c|c|}
\hline \multicolumn{2}{|l|}{$\mathrm{A}$} & \multicolumn{2}{|l|}{ B } \\
\hline$B_{0} / \mathrm{mT}$ & $T_{1 \mathrm{e}}\left(\Delta_{i}\right) / \mathrm{ms}$ & $B_{0} / \mathrm{mT}$ & $T_{1 \mathrm{e}}\left(\Delta_{i}\right) / \mathrm{ms}$ \\
\hline 3344.3 & 124 & 3341.7 & 5 \\
\hline 3348.0 & 30 & 3345.0 & 11 \\
\hline 3351.7 & 46 & 3348.2 & 6 \\
\hline 3355.4 & 127 & 3351.5 & 13 \\
\hline \multirow[t]{2}{*}{3359.1} & 272 & 3354.8 & 30 \\
\hline & & 3358.1 & 79 \\
\hline
\end{tabular}

that is effectively averaged, while infinitely long relaxation times would be averaged over the entire spectrum. Yet, for our samples, we experimentally determined anisotropic $T_{1 \mathrm{e}}(\omega)$ values even for $50 \mathrm{mM}$ TEMPOL at W-band at $6 \mathrm{~K}(94.1 \mathrm{GHz}$; centre field $3.35 \mathrm{~T}$; vide supra) showing that the polarization is not completely averaged by eSD across the entire EPR linewidth in the absence of continuous microwave irradiation. This is in agreement with earlier findings by Farrar et al. ${ }^{14}$

It has been pointed out by Wenckebach ${ }^{8}$ that eSD is strongly temperature and field dependent, so that it is difficult to make any reasonable assumptions for $6.7 \mathrm{~T}$ and $4 \mathrm{~K}$. Yet, using eSD coefficients recently estimated by Siaw et al. ${ }^{28}$ and the $T_{1 \mathrm{e}}(\omega)$ values reported in Table 1, we can approximate the fractions of the EPR spectrum that are averaged by eSD via the formalism described in Wenckebach's monograph (chapter 5.3). ${ }^{8}$ We assume a frequency $\omega_{0}$, with an offset to the centre of gravity $\omega_{\mathrm{e}}$ of the EPR line is $\Delta_{0}=\omega_{0}-\omega_{\mathrm{e}}$. In the case of DNP, when a spin packet at an offset $\Delta_{0}$ is saturated, the microwave field also disturbs the $i$ th electron spin packet at an offset $\Delta_{i}=\omega_{i}-\omega_{\mathrm{e}}$. Accordingly, we determined the contribution of a spin packet $i$ to the electronic relaxation $T_{1 \mathrm{e}}(\omega)$ measured at an offset $\Delta_{0}$ by $f\left(\Delta_{i}\right) \exp \left(\left(\Delta_{i}-\Delta_{0}\right) /\right.$ $\left(D T_{1 \mathrm{e}}\left(\Delta_{i}\right)\right)^{1 / 2}$ ) where $D$ denotes the spectral diffusion coefficient and where the frequency $\omega$ has been replaced by the offset $\Delta_{i}$ to yield $T_{1 \mathrm{e}}\left(\Delta_{i}\right)$. We denote the normalized amplitude of the absorption EPR spectrum by $f\left(\Delta_{i}\right)$. If the width of the EPR spectrum is $M^{2}$, the spectral diffusion coefficient is $D=M^{2} / 4 \tau_{\mathrm{SD}}$. Since $\tau_{\mathrm{SD}}$ can be estimated to be $10 \mathrm{~ms},{ }^{28}$ only part of the EPR spectrum is effectively smoothed by eSD at the concentrations used in our DNP experiments.

The spin packets of the EPR spectrum that are graphically depicted in Fig. 4 represent contributions to the detected $T_{1 \mathrm{e}}\left(\Delta_{i}\right)$ values. Note that significant spin packets of the spectrum are averaged, though not across the entire spectrum, thus making it possible for $T_{1 \mathrm{e}}\left(\Delta_{i}\right)$ to remain anisotropic. In the presence of a CW microwave field, it has further to be considered that eSD coefficients change as the polarization of the individual spin packets varies and $D \propto\left(1-P_{\mathrm{e}}^{2}\right)$. Recent double-electron resonance experiments, ${ }^{15}$ yet, show that even under such conditions the EPR line is not entirely averaged via eSD for our experimental setup (6.7 T, 4 K, 25 mM TEMPOL). Furthermore, the spectral diffusion rate might also depend on the amplitude of the EPR spectrum at a particular field/frequency, a fact that also affects the anisotropy of $T_{1 \mathrm{e}}\left(\Delta_{i}\right)$ relaxation, since eSD-induced averaging is much stronger near the centre of gravity of the spectrum than at its extremities. Indeed, the observed relaxation time $T_{1 \mathrm{e}}\left(\Delta_{i}\right)$ is a weighted average across the spectral range covered by eSD, as demonstrated in Fig. 4. Hence, the faster eSD, the more packets will contribute to each $T_{1 \mathrm{e}}\left(\Delta_{i}\right)$ value, so that these will appear more isotropic if spectral diffusion is fast. On the contrary, for slow eSD, the anisotropy of $T_{1 \mathrm{e}}\left(\Delta_{i}\right)$ can be more pronounced. Thus, $T_{1 \mathrm{e}}\left(\Delta_{i}\right)$ values detected near the centre of the EPR spectrum can be less anisotropic then those observed near the edges of the spectrum. Such a behaviour can be observed in Fig. 3, where the strongest deviations from the average relaxation time can be observed near the edges of the EPR spectrum.

\section{Dynamic nuclear polarization}

For the case at hand, we consider a mono-radical with a broad EPR spectrum at low temperatures, with a width that is comparable to the proton nuclear Larmor frequency, which is near $285 \mathrm{MHz}$ in our experiments. To demonstrate how anisotropic 

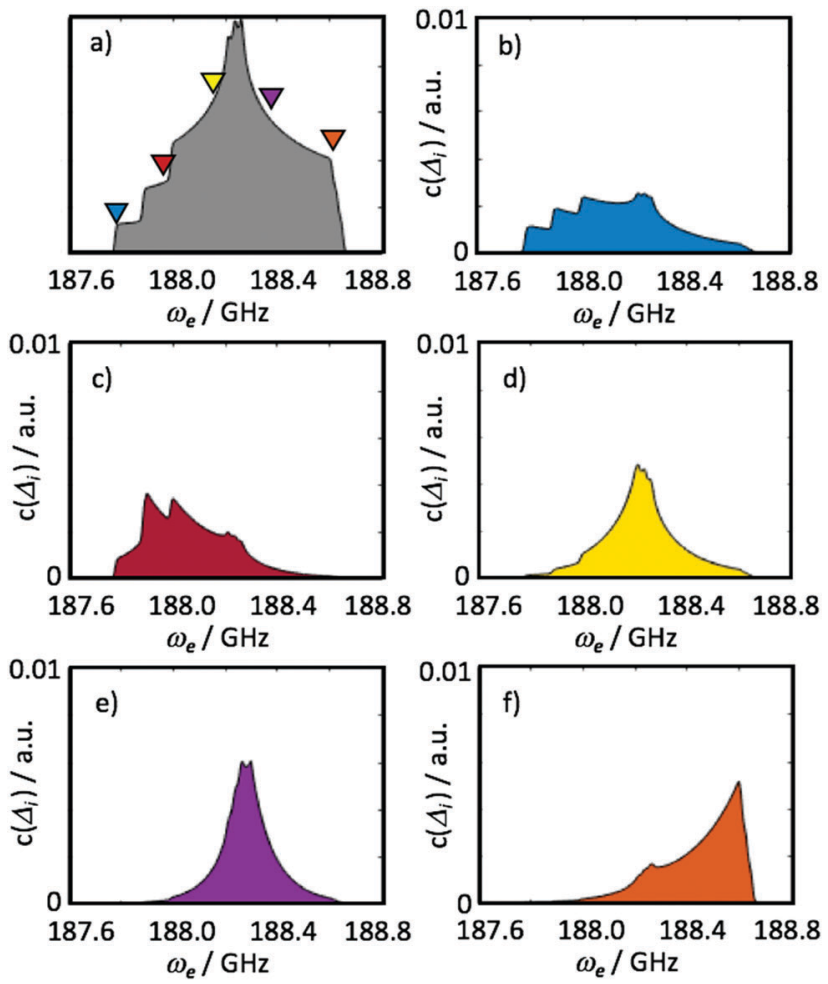

Fig. 4 (a) Simulated EPR spectrum at $6.7 \mathrm{~T}$ with $N=5$ different $T_{1 \mathrm{e}}\left(\Delta_{i}\right)$ measured at the frequencies indicated by color codes. Note that the eSD rates could not be determined experimentally. (b-f) Simulated contributions $c\left(\Delta_{i}\right)$ of different spin packets to the apparent $T_{1 \mathrm{e}}\left(\Delta_{i}\right)$ measured at five different positions. The coefficients are normalized so that $\sum\left[c\left(\Delta_{i}\right)\right]=1$.

$T_{1 \mathrm{e}}\left(\Delta_{i}\right)$ can be incorporated into a model describing DNP at low temperatures, we chose Borghini's theory for the sake of simplicity. Based on strong assumptions, i.e., a single spin temperature and a strong contact between the nuclear Zeeman and electronic dipolar bath, this model yields simple predictions, which can easily be adapted to anisotropic $T_{1 \mathrm{e}}\left(\Delta_{i}\right)$ although the model assumes fast eSD to justify the use of a single spin temperature. Borghini's model has been reviewed in depth by Abragam and Goldman ${ }^{1}$ using a thermodynamic description. Jannin et al. pointed out that Borghini's approach corresponds to a Redfield model with a single spin temperature in the rotating frame. ${ }^{29}$ It can be regarded as an extension of Provotorov's theory to low temperatures, since one can predict the temperature of the non-Zeeman reservoir for off-centre saturation of the EPR line by dissecting the latter into several spin packets.

We briefly outline Borghini's theory. ${ }^{16}$ At equilibrium, the polarization $P_{0}$ of the electrons is given by a Boltzmann distribution:

$$
\begin{gathered}
\left|P_{0}\right|=\tanh \left(\frac{1}{2} \beta_{\mathrm{L}} \omega_{\mathrm{e}} \hbar\right) \\
\beta_{\mathrm{L}}=\frac{1}{k_{\mathrm{B}} T_{\mathrm{L}}}
\end{gathered}
$$

where $\omega_{\mathrm{e}}, T_{\mathrm{L}}, k_{\mathrm{B}}$ are the electron Larmor frequency, the lattice temperature and the Boltzmann constant, whilst $\beta_{\mathrm{L}}$ denotes the inverse temperature coefficient of the lattice.
For the case at hand, the line width of the EPR spectrum is dominated by a spread (anisotropy) of the electronic $g$ factor at the high magnetic field of $6.7 \mathrm{~T}$. It is possible to relate the nuclear polarization to the EPR line shape if we consider electrons at frequency $\Delta_{0}$ to be fully saturated ( $c f$. Fig. 1b). Under these conditions, $P_{\mathrm{e}}\left(\Delta_{0}\right)=0$, while the polarization $P_{\mathrm{e}}\left(\Delta_{i}\right)$ of a spin packet at an offset $\Delta_{i}$ is $P_{\mathrm{e}}\left(\Delta_{i}\right)=-\tanh \left[\frac{1}{2} \beta\left(\Delta_{0}-\Delta_{i}\right) \hbar\right]$, where $\beta$ is the electron dipolar (non-Zeeman) inverse temperature coefficient, so that spin packets located at a frequency offset $\Delta_{i}$ are partially saturated and contribute to DNP. If the electronic $g$ anisotropy dominates the spread of the electron frequencies, the electron polarization affects the steady-state nuclear polarization $P_{\mathrm{n}}$ according to Borghini: ${ }^{1}$

$$
\left.P_{\mathrm{n}}=\frac{T_{1 \mathrm{n}} C}{\omega_{\mathrm{n}} T_{\mathrm{le}}} \sum_{i} f\left(\Delta_{i}\right)\left[\left(\Delta_{0}-\Delta_{i}\right) P_{\mathrm{e}}\left(\Delta_{i}\right)+\Delta_{0} P_{0}\right]\right)
$$

Here $\omega_{\mathrm{n}}$ is the nuclear Larmor frequency and $C=N_{\mathrm{e}} / N_{\mathrm{n}}$ denotes the ratio of the number of electron spins to the number of nuclear spins. $T_{1 \mathrm{e}}$ and $T_{1 \mathrm{n}}$ are the electron and nuclear longitudinal relaxation times, respectively, both of which are considered to be isotropic in Borghini's original model. The steady-state value of the dipolar (non-Zeeman) inverse temperature coefficient of the electrons $\beta_{0}$ under $\mu \mathrm{w}$ irradiation at the offset $\Delta_{0}$ can be expressed by eqn (4) and (5):

$$
\begin{gathered}
\beta_{0}=\frac{2 \Delta_{0}\left|P_{0}\right|}{\left(\Delta_{0}^{2}+D^{2}\right)}=\frac{1}{k_{\mathrm{B}} T_{\mathrm{S}}} \\
M^{2}=\int_{-\infty}^{+\infty} \Delta^{2} f(\Delta) \mathrm{d} \Delta
\end{gathered}
$$

Here $M^{2}$ is the second moment of the EPR line, $P_{0}$ the thermal electronic polarization and $T_{\mathrm{s}}$ is the spin temperature.

To account for the anisotropy of the longitudinal electronic relaxation times that vary across the EPR line, we introduce an offset-dependent $T_{1 \mathrm{e}}\left(\Delta_{i}\right)$. To this end, we adapt the following rate equations derived by Abragam and Goldman: ${ }^{1}$

$$
\left.\frac{\mathrm{d}}{\mathrm{d} t}\left\langle\mathcal{H}_{\mathrm{Ze}}\right\rangle=\frac{N_{\mathrm{e}}}{2 \omega_{\mathrm{e}}} \sum_{i} \frac{f\left(\Delta_{\mathrm{i}}\right)}{T_{1 \mathrm{e}}}\left[P_{\mathrm{e}}\left(\Delta_{i}\right)-P_{0}\right]+U f_{0} P_{\mathrm{e} 0}\right)
$$

and

$$
\left.\frac{\mathrm{d}}{\mathrm{d} t}\left\langle\mathcal{H}_{\mathrm{Zn}}+\mathcal{H}_{\mathrm{nZ}}\right\rangle=\frac{N_{\mathrm{e}} P_{\mathrm{n}}}{T_{1 \mathrm{n}} 2 C \omega_{\mathrm{n}}}+\frac{N_{\mathrm{e}}}{2} \sum_{i} \frac{f\left(\Delta_{i}\right)}{T_{1 \mathrm{e}}} P_{\mathrm{eI}}+U f_{0} \Delta_{0} P_{0}\right)
$$

where $\mathcal{H}_{\mathrm{Ze}}, \mathcal{H}_{\mathrm{Zn}}$ and $\mathcal{H}_{\mathrm{nZ}}$ denote the electron-Zeeman, nuclearZeeman and non-Zeeman Hamiltonians. $P_{\mathrm{e} 0}$ is the polarization of the saturated packet $\Delta_{0} . N_{\mathrm{e}}$ is the number of electron spins. Replacing $T_{1 \mathrm{e}}$ by $T_{1 \mathrm{e}}\left(\Delta_{i}\right)$ and setting the derivatives to zero to describe the steady state, we can derive a modified Borghini equation where terms $T_{1 \mathrm{e}}\left(\Delta_{i}\right)$ remain within the summation. 
Combining eqn (6) and (7) leads to:

$$
\left.P_{\mathrm{n}}=\frac{T_{\mathrm{ln}} C}{\omega_{\mathrm{n}}} \sum_{i} \frac{f\left(\Delta_{i}\right)\left(\Delta_{0}-\Delta_{i}\right)}{T_{1 \mathrm{e}}\left(\Delta_{i}\right)}\left[P_{\mathrm{e}}\left(\Delta_{i}\right)-P_{0}\right]\right)
$$

Note that for nitroxides in partially deuterated glassy matrices, the width of EPR spectra at $6.7 \mathrm{~T}$ is primarily determined by the $g$-anisotropy and by intramolecular hyperfine couplings. However, the presence of additional contributions to homogeneous and heterogeneous line broadening cannot be entirely excluded $a$ priori. Indeed, intermolecular dipolar electron-electron couplings can contribute to the width of the EPR spectra, especially at the high nitroxide concentrations that are typically used for DNP.

In conclusion, since the nuclear polarization $P_{\mathrm{n}}$ in eqn (8) depends on the various $T_{1 \mathrm{e}}\left(\Delta_{i}\right)$ contributions, which can vary significantly even after averaging through eSD, the observed proton signal intensity will also vary across the microwave profile.

The incorporation of an offset-dependent $T_{1 \mathrm{e}}\left(\Delta_{i}\right)$ for different spin packets is of importance as the bottleneck of the spin dynamics is influenced by $T_{1 \mathrm{e}}\left(\Delta_{i}\right)$. In other words, the nuclear polarization that can be reached in each spin packet is directly related to $T_{1 \mathrm{e}}\left(\Delta_{i}\right)$ as pointed out by Serra et al. ${ }^{13}$ Hence, the dependence of $P_{\mathrm{n}}$ on $T_{1 \mathrm{e}}\left(\Delta_{i}\right)$ must be considered to predict the shape of a DNP profile if eSD is sufficiently slow such that $T_{1 \mathrm{e}}\left(\Delta_{i}\right)$ varies with the orientations of the $g$-tensors for different spin packets. As the Borghini model neglects the anisotropy of $T_{1 \mathrm{e}}\left(\Delta_{i}\right)$, since it assumes fast eSD underlying TM this leads to a bias in the frequency dependence of the predicted nuclear polarization.

More generally, any model that takes into account the whole EPR line profile must also address the frequency dependence of the electron spin-lattice relaxation times $T_{1 \mathrm{e}}\left(\Delta_{i}\right)$ within that line adequately.

Borghini's model does not describe all details of nitroxidebased DNP. In particular, absolute polarization levels cannot be predicted correctly, as the requirement of a single spin temperature is often not fulfilled under practical conditions. Nonetheless, the match between theory and experiment can be significantly improved by incorporating anisotropic relaxation properties.

More sophisticated models such as those presented by Vega and co-workers, ${ }^{9-12,15,18,24-27,30}$ which account for subtle aspects of DNP (e.g., temperature- and concentration-dependence, absolute polarization), could presumably be improved by taking into account the anisotropy of electronic relaxation.

Fig. 5 displays "DNP enhancement profiles", i.e., the normalized steady-state integrated proton signal intensity observed at $4.2 \mathrm{~K}$ (after complete build-up or extrapolated to the steady-state; $c f$. Fig. 1) as a function of the microwave frequency in our DNP apparatus. After normalization, the ${ }^{1} \mathrm{H}$ signal intensity corresponds to $P_{\mathrm{n}}$ in eqn (8). The data shown in Fig. $5 \mathrm{a}$ and b were obtained for samples A and B, both of which contain $25 \mathrm{mM}$ TEMPOL but in different solvents ( $c f$. Experimental). Calculated enhancement profiles based on eqn (8) are superimposed as solid lines, assuming that the simulated (based on experimentally determined $g$ - and $A$-tensors plus an additional linewidth)
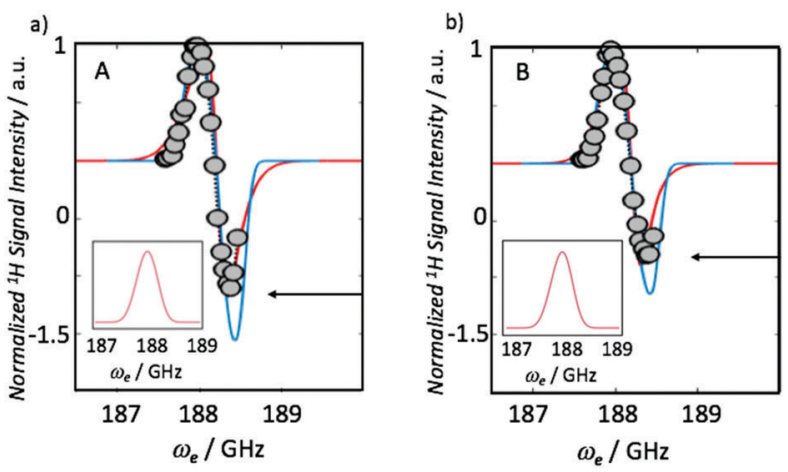

Fig. 5 (a) Normalized steady-state proton signal intensity as a function of the microwave frequency $\omega_{\mathrm{e}}$ ("DNP enhancement profile") for $25 \mathrm{mM}$ TEMPO at $4.2 \mathrm{~K}$ : (a) in glycerol- $\mathrm{d}_{8} / \mathrm{D}_{2} \mathrm{O} / \mathrm{H}_{2} \mathrm{O}(5: 4: 1)$; (b) in ethanol- $\mathrm{d}_{6} /$ ethanol $(9: 1)$. Circles indicate experimentally determined steady-state polarizations, solid red lines the calculated responses. The inserts display the simulated EPR spectra underlying the calculations. Pronounced differences in the negative lobes are emphasized by arrows. The blue lines indicate DNP profiles based on eqn (3) obtained for a single isotropic relaxation time $T_{1 \mathrm{e}}$ that does not depend on $\Delta_{i}$.

EPR spectra (see insets) are broadened in accordance with the influence of eSD.

If the polarity of the solvent is modified, changes in the DNP enhancement profiles are observed. For TEMPOL dissolved in ethanol (sample B in Fig. 5b), the observed changes with respect to more polar environment of sample A can be modelled by variations of the anisotropic $T_{1 \mathrm{e}}\left(\Delta_{i}\right)$ (recalling that the $g$ - and $A$-tensors were determined experimentally, vide supra). When switching from sample A to B, the DNP efficiency of the highfield lobe is significantly reduced. The shape of the DNP profile can be modelled by eqn (8), if one considers the anisotropy of $T_{1 \mathrm{e}}\left(\Delta_{i}\right)$ documented in Table 1 . Although variations in solvent polarity can lead to variations in local microscopic environments and hence to variations of nitroxide $g$ - and $A$-tensors, we find that the calculations of Fig. 5 need not to invoke such effects, which can be neglected here due to the strong line broadening at high TEMPOL concentrations. ${ }^{31}$

The solid blue lines in Fig. 5 clearly show that the shape and relative lobe magnitudes of the experimental DNP profiles cannot be reproduced if only a single frequency-independent relaxation time $T_{1 \mathrm{e}}$ is considered. Changing the EPR line width (which is the only adaptable parameter) cannot account for the discrepancies. Obviously, the match between the experimental data and theoretical prediction is significantly improved by considering anisotropic relaxation times $T_{1 \mathrm{e}}\left(\Delta_{i}\right)$.

\section{Methodological considerations}

Several points must be critically considered when using eqn (8):

(i) $T_{1 \mathrm{e}}\left(\Delta_{i}\right)$ varies significantly with the $\mu \mathrm{w}$ offset as indicated in Fig. 3 and Table 1. To obtain a continuous fit (Fig. 5), we measured $T_{1 \mathrm{e}}\left(\Delta_{i}\right)$ at low concentrations and hence slow eSD with selective pulses to obtain values that reflect the $T_{1 \mathrm{e}}\left(\Delta_{i}\right)$ of a narrow spin packet of the EPR spectrum and interpolated $T_{1 \mathrm{e}}\left(\Delta_{i}\right)$ between the observed data points using 4 th and 5 th-order polynomials for samples A and B respectively ( $c f$. Fig. 5) to account for 
the averaging through eSD. Because of the sparse $T_{1 \mathrm{e}}\left(\Delta_{i}\right)$ measurements, abrupt changes in $T_{1 \mathrm{e}}\left(\Delta_{i}\right)$ might have been overlooked. Nevertheless, the match is improved between the experimental DNP profiles and those predicted via this approach.

It was further assumed that changes of $T_{1 \mathrm{e}}\left(\Delta_{i}\right)$ between $6 \mathrm{~K}$ (where $T_{1 \mathrm{e}}\left(\Delta_{i}\right)$ was measured) and $4.2 \mathrm{~K}$ (where the DNP enhancement profiles were obtained) are uniform across the spectrum and do not depend on the offset $\Delta_{0}$, so that normalization of the DNP profiles compensates for a possible temperature dependence (we excluded strong field dependence, vide supra).

(ii) The solution of eqn (8) and the fits of the enhancement profiles in Fig. 5 require prior knowledge of the EPR spectra $f\left(\Delta_{i}\right)$. These spectra were obtained via simulation as illustrated in Fig. 2. (Note, however, that the EPR spectrum was determined at a TEMPOL concentration of $1 \mathrm{mM}$, whereas DNP was performed at $25 \mathrm{mM}$. The spectra should thus not be compared directly.) After normalization, the only freely adjustable parameter in eqn (8) is the EPR line width (since the $g$ - and $A$-tensors of the EPR spectrum have been determined experimentally, vide supra). The EPR line width cannot account for the discrepancy between experimental data and the theoretical predictions obtained via eqn (3), i.e., for the case of isotropic relaxation times $T_{1 \mathrm{e}}$ that do not depend on $\Delta_{0}$.

Further, spectral diffusion mixes the on-resonance spin packet saturated by $\mu \mathrm{w}$ irradiation at frequency $\omega_{0}$ with spin packets at other frequencies. Thus, DNP-determined spectra at $6 \mathrm{~K}$ and $25 \mathrm{mM}$ typically appear strongly broadened ${ }^{32}$ since at each EPR frequency the intensity is averaged by spectral diffusion. This is taken into account by superimposing an additional linewidth on the spectrum shown in Fig. 2 b.

(iii) Borghini's model does not consider possible effects of non-uniform spectral diffusion coefficients on the EPR line shape. Spectral diffusion is only accounted for by assuming a rapid equilibration over the entire EPR spectrum.

One might therefore speculate that anisotropic spectral diffusion and line broadening could likewise account for discrepancies between the model and the experiments, and should therefore also be incorporated into more sophisticated models. For the case at hand we did not include such eSD effects or line width parameters in the Borghini model, which was in this work mainly used to emphasize the importance of $T_{1 \mathrm{e}}\left(\Delta_{i}\right)$ anisotropy. Note, however, that the simulated EPR spectrum is in agreement with spectra obtained under comparable conditions ${ }^{32}$ and was kept constant in all our calculations.

(iv) Borghini's TM model is only valid when the on-resonance spin packet is fully saturated and when the thermal contact between the electronic dipolar and nuclear Zeeman reservoirs is strong. Although we verified that the on-resonance electrons were fully saturated (see Fig. 1), the Borghini model (even after incorporating anisotropic electronic relaxation) still overestimates $P_{\mathrm{n}}$ at the extremities of the profile, as the irradiated spin packet is only a small fraction of the ensemble and the contact with the nuclear bath is therefore weaker than assumed in the model. ${ }^{33}$ Other more sophisticated models might be capable of compensating for such flaws. Especially, recent work by Wenckebach ${ }^{34}$ extends the theoretical description of TM
DNP to low temperatures, overcoming limitations of Borghini's model by predicting the polarization of off-resonance electron spins. However, the focus of this work is not to propose a new model to describe DNP, but to draw attention to the importance of anisotropic electronic relaxation. Its incorporation into virtually any physical model should improve the agreement between predicted and experimental DNP profiles if spectral diffusion is slow.

\section{Conclusions}

Our data suggest that DNP models should be refined by incorporating the anisotropy of electronic longitudinal relaxation times, since at low temperatures and high magnetic fields $T_{1 \mathrm{e}}\left(\Delta_{i}\right)$ significantly depends on the molecular orientation if electronic spectral diffusion does not lead to full averaging of the polarization across the entire EPR spectrum. ${ }^{15}$

This amendment seems quite reasonable in view of our measurements of the frequency-dependence of $T_{1 \mathrm{e}}\left(\Delta_{i}\right)$, corresponding to a dependence on the molecular orientation. The variations of $T_{1 \mathrm{e}}\left(\Delta_{i}\right)$ can be neglected at lower fields (e.g., at X-band at $0.3 \mathrm{~T})$, but at $\mathrm{W}$-band $(94.1 \mathrm{GHz}$, centre field $3.5 \mathrm{~T})$, and a fortiori at $6.7 \mathrm{~T}$ (centre frequency $188.2 \mathrm{GHz}$ ) where our DNP experiments have been carried out, the anisotropy of $T_{1 \mathrm{e}}\left(\Delta_{i}\right)$ cannot be neglected.

While the extension of Borghini's model introduced here can reproduce the main features of our DNP profiles it will certainly not adequately describe all the subtleties of DNP at cryogenic temperatures. Yet, we show that even the rather rudimentary Borghini model can be amended to take our observations into account. Clearly, anisotropic electronic relaxation should be included in all models describing nitroxide-based DNP. Indeed, to the best of our knowledge, all known models indicate that the proton polarization depends on $T_{1 \mathrm{e}}\left(\Delta_{i}\right)$.

\section{Acknowledgements}

The authors acknowledge Bruker BioSpin for generously providing state-of-the-art D-DNP equipment. The authors want to thank Prof. Gunnar Jeschke and Dr Maxim Yulikov for providing access to the W-band EPR spectrometer, Prof. Konstantin L. Ivanov for stimulating discussions, and Dr Alberto Rosso for helpful discussions and the derivation of eqn (8). This work was supported by the CNRS's infrastructure RENARD (FR3443) for EPR facilities and the European Research Council (ERC contract 'Dilute para-water').

\section{Notes and references}

1 A. Abragam and M. Goldman, Rep. Prog. Phys., 1978, 41(3), 395-467.

2 D. Kurzbach, E. M. M. Weber, A. Jhajharia, S. F. Cousin, A. Sadet, S. Marhabaie, E. Canet, N. Birlirakis, J. Milani, S. Jannin, D. Eshchenko, A. Hassan, R. Melzi, S. Luetolf, M. Sacher, M. Rossire, J. Kempf, J. A. B. Lohman, M. Weller, 
G. Bodenhausen and D. Abergel, J. Chem. Phys., 2016, 145, 194203.

3 H. Min, G. Sekar and C. Hilty, ChemMedChem, 2015, 10(9), 1559-1563.

4 S. Jannin, A. Bornet, S. Colombo and G. Bodenhausen, Chem. Phys. Lett., 2011, 517(4-6), 234-236.

5 J. H. Ardenkjaer-Larsen, B. Fridlund, A. Gram, G. Hansson, L. Hansson, M. H. Lerche, R. Servin, M. Thaning and K. Golman, Proc. Natl. Acad. Sci. U. S. A., 2003, 100(18), 10158-10163.

6 D. Kurzbach, E. Canet, A. G. Flamm, A. Jhajharia, E. M. Weber, R. Konrat and G. Bodenhausen, Angew. Chem., Int. Ed. Engl., 2017, 56(1), 389-392.

7 A. Jhajharia, E. M. Weber, J. G. Kempf, D. Abergel, G. Bodenhausen and D. Kurzbach, J. Chem. Phys., 2017, 146(4), 041101.

8 T. Wenckebach, Essentials of Dynamic Nuclear Polarization, Spindrift Publications, 2016.

9 Y. Hovav, A. Feintuch and S. Vega, J. Magn. Reson., 2010, 207(2), 176-189.

10 Y. Hovav, O. Levinkron, A. Feintuch and S. Vega, Appl. Magn. Reson., 2012, 43(1-2), 21-41.

11 Y. Hovav, A. Feintuch and S. Vega, J. Magn. Reson., 2012, 214, 29-41.

12 Y. Hovav, A. Feintuch and S. Vega, Phys. Chem. Chem. Phys., 2013, 15(1), 188-203.

13 S. C. Serra, A. Rosso and F. Tedoldi, Phys. Chem. Chem. Phys., 2012, 14(38), 13299-13308.

14 C. T. Farrar, D. A. Hall, G. J. Gerfen, S. J. Inati and R. G. Griffin, J. Chem. Phys., 2001, 114(11), 4922-4933.

15 A. Leavesley, D. Shimon, T. A. Siaw, A. Feintuch, D. Goldfarb, S. Vega, I. Kaminker and S. Han, Phys. Chem. Chem. Phys., 2017, 19(5), 3596-3605.

16 M. Borghini, Phys. Rev. Lett., 1968, 20(9), 419-421.

17 A. Bornet, J. Milani, B. Vuichoud, A. J. P. Linde, G. Bodenhausen and S. Jannin, Chem. Phys. Lett., 2014, 602, 63-67.
18 Y. Hovav, A. Feintuch, S. Vega and D. Goldfarb, J. Magn. Reson., 2014, 238, 94-105.

19 F. Delaglio, S. Grzesiek, G. W. Vuister, G. Zhu, J. Pfeifer and A. Bax, J. Biomol. NMR, 1995, 6(3), 277-293.

20 S. Stoll and A. Schweiger, Biol. Magn. Reson., 2007, 27, 299-321. 21 S. Stoll and A. Schweiger, J. Magn. Reson., 2006, 178(1), 42-55.

22 M. F. Ottaviani, M. Garciagaribay and N. J. Turro, Colloids Surf., A, 1993, 72, 321-332.

23 V. A. Atsarkin, A. E. Mefeod and M. I. Rodak, Phys. Lett. A, 1968, 27(1), 57-\&.

24 Y. Hovav, D. Shimon, I. Kaminker, A. Feintuch, D. Goldfarb and S. Vega, Phys. Chem. Chem. Phys., 2015, 17(8), 6053-6065.

25 Y. Hovav, I. Kaminker, D. Shimon, A. Feintuch, D. Goldfarb and S. Vega, Phys. Chem. Chem. Phys., 2015, 17(1), 226-244.

26 D. Shimon, Y. Hovav, A. Feintuch, D. Goldfarb and S. Vega, Phys. Chem. Chem. Phys., 2012, 14(16), 5729-5743.

27 A. Feintuch, D. Shimon, Y. Hovav, D. Banerjee, I. Kaminker, Y. Lipkin, K. Zibzener, B. Epel, S. Vega and D. Goldfarb, J. Magn. Reson., 2011, 209(2), 136-141.

28 T. A. Siaw, M. Fehr, A. Lund, A. Latimer, S. A. Walker, D. T. Edwards and S. I. Han, Phys. Chem. Chem. Phys., 2014, 16(35), 18694-18706.

29 S. Jannin, A. Comment, F. Kurdzesau, J. A. Konter, P. Hautle, B. van den Brandt and J. J. van der Klink, J. Chem. Phys., 2008, 128(24), 241102.

30 D. Shimon, A. Feintuch, D. Goldfarb and S. Vega, Phys. Chem. Chem. Phys., 2014, 16(14), 6687-6699.

31 D. Kurzbach, M. J. N. Junk and D. Hinderberger, Macromol. Rapid Commun., 2013, 34(2), 119-134.

32 P. Niedbalski, C. Parish, A. Kiswandhi and L. Lumata, Magn. Reson. Chem., 2016, 54(12), 962-967.

33 S. C. Serra, A. Rosso and F. Tedoldi, Phys. Chem. Chem. Phys., 2013, 15(21), 8416-8428.

34 W. T. Wenckebach, J. Magn. Reson., 2017, 277, 68-78.

35 J. L. Du, G. R. Eaton and S. S. Eaton, J. Magn. Reson., Ser. A, 1995, 115, 213-221. 